\title{
Diffuse Astrocytoma Initially Presenting as a Massive Intracerebral Hemorrhage: Case Report
}

\author{
Takao NOZAKI, ${ }^{1}$ Haruhiko SATO, ${ }^{2}$ Tomohiro YAmAZOE, ${ }^{1}$ and Hiroki NAMBA ${ }^{1}$ \\ ${ }^{1}$ Department of Neurosurgery, Hamamatsu University School of Medicine, \\ Hamamatsu, Shizuoka; \\ ${ }^{2}$ Department of Neurosurgery, Seirei Mikatahara General Hospital, Hamamatsu, Shizuoka
}

\begin{abstract}
We report the case of a 58-year-old woman with low-grade astrocytoma, who developed massive intracranial hemorrhage as the first presentation of this disease, and become comatose and subsequently underwent an emergency craniotomy. A small amount of tumor-like tissue was observed on the wall of the hematoma cavity. Histological analysis of the resected specimen indicated diffuse astrocytoma [World Health Organization (WHO) grade II]. The patient was discharged without neurological deficits 2 weeks after the operation. A non-enhanced tumor-like nodule was observed on magnetic resonance imaging 3 months after the operation, which was monitored carefully but was not treated by adjuvant therapy. The tumor grew gradually, and a second operation was performed 3 years after the first, in which the tumor was completely resected. Histological analysis of the resected specimen again indicated diffuse astrocytoma (WHO grade II). Although rare, brain tumors, including low-grade astrocytoma, should be considered a possible cause of subcortical hemorrhage in patients without risk factors for intracranial hemorrhage.
\end{abstract}

Key words: low-grade astrocytoma, diffuse astrocytoma, hemorrhage, stroke

\section{Introduction}

Brain tumors are an important cause of intracerebral hemorrhage. The incidence of intratumoral hemorrhage in gliomas is reported to be $3.7-12 \%$, and the likelihood of hemorrhage in these cases depends mainly on the tumor histology. Intratumoral hemorrhages usually occur in high-grade gliomas and rarely in low-grade ones $(<1 \%)$. A massive hematoma as an initial manifestation of lowgrade astrocytoma is also extremely rare. All reported cases of intratumoral hemorrhage have been observed in cases of pilocytic astrocytomas, ${ }^{1-3)}$ and no previous study has reported this type of hemorrhage in cases of diffuse astrocytoma. Here we report a case of diffuse astrocytoma manifesting as a large and expansive subcortical hemorrhage mimicking a stroke.

\section{Case Report}

A 58-year-old woman with a sudden onset of headache was admitted in a somnolent state, without focal signs. Computed tomography (CT) scan revealed a massive

Received May 31, 2013; Accepted August 15, 2013 subcortical hemorrhage in the right frontal lobe, mimicking a stroke (Fig. 1). Enhanced CT scan and digital subtraction angiography (DSA) did not indicate abnormal vessels or tumor stains (not shown). The patient had neither a history of hypertension nor a bleeding tendency. After undergoing the imaging procedures, she became comatose and subsequently an emergency craniotomy was performed. A small amount of tumor-like tissue was observed on the wall of the hematoma cavity. Histological analysis of the resected specimen indicated diffuse astrocytoma [World Health Organization (WHO) grade II; Fig. 1]. Two weeks postoperatively, the patient was discharged without neurological deficits. Magnetic resonance imaging (MRI) scan 3 months after the operation revealed a small residual tumor on the hematoma cavity (Fig. 2), which was monitored carefully although no adjuvant therapy was administered.

The tumor grew gradually (Fig. 3), and a second operation was performed 3 years after the first, in which the tumor was completely resected. Histological diagnosis of the resected specimens again revealed a diffuse astrocytoma [WHO grade II, GFAP+, IDH1+, p53 mutation+, mindbomb homolog 1 (MIB-1) labeling index: 4-5\%; Fig. 3C]. Postoperative irradiation was delivered to the right frontal lobe. 


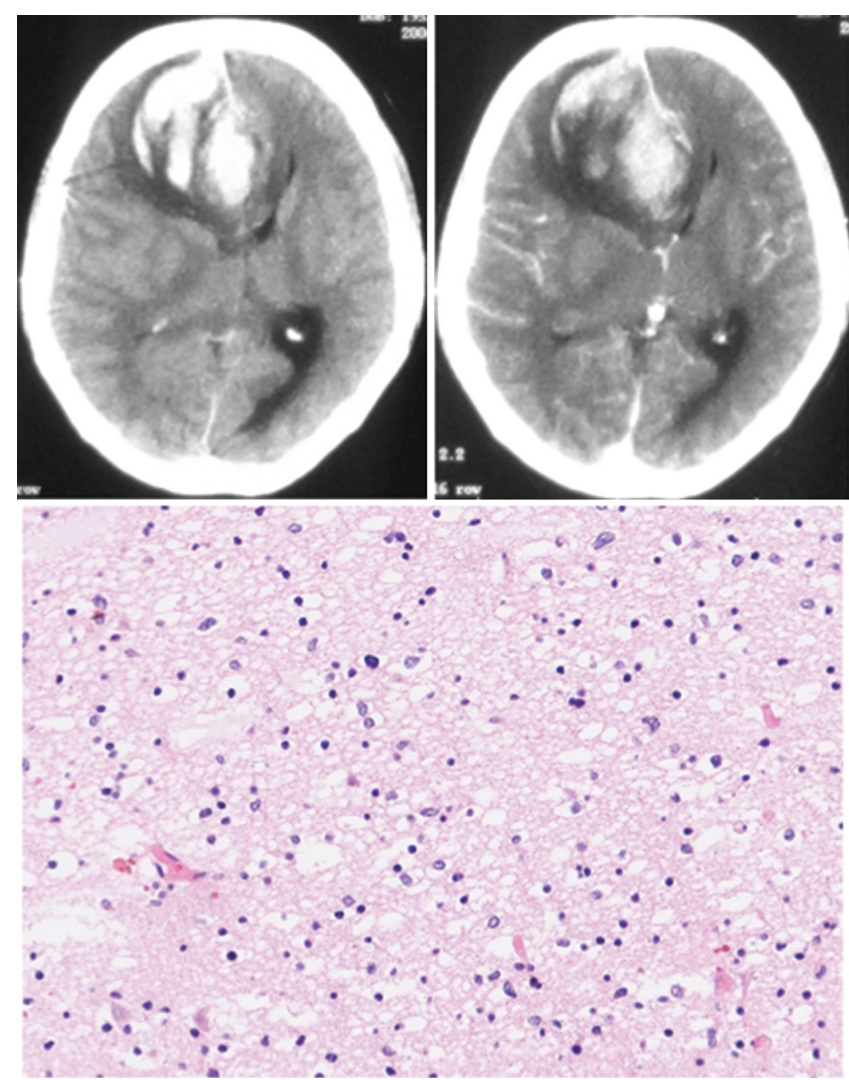

Fig. 1 A pre-contrast enhanced computed tomography (CT) scan showing a massive subcortical hematoma in the right frontal lobe (top, left). A post-contrast enhancement CT scan showing the absence of abnormal enhancement arround the hematoma (top, right). Histological examination of the resected tumor specimen showing the features of diffuse astrocytoma [bottom $\times 200$, hematoxylin and eosin stain (HE)].

\section{Discussion}

Low-grade astrocytoma with an initial presentation of intracranial hemorrhage is extremely rare, with only 16 reports (20 cases) in the literature, and all hemorrhages have been reported in patients with pilocytic or pilomyxoid tumors. ${ }^{1-5)}$ Of these 16 reports, only 3 cases presented with a massive and stroke-like hematoma. ${ }^{1-3)}$ This is the first reported case of diffuse astrocytoma presenting with a massive hemorrhage. All the hematomas associated with low-grade astrocytomas, including the one observed in the present case, were located in the subcortical region. The diagnosis of this tumor-associated hematoma is difficult. The tumor could not be detected by preoperative CT scan (with or without contrast enhancement) or by DSA. MRI, which was not performed preoperatively because of the patient's neurological deterioration, would have aided tumor detection. We suggest that a careful postoperative follow-up with MRI is indispensable for subcortical hematomas in patients without risk factors of hemorrhage.

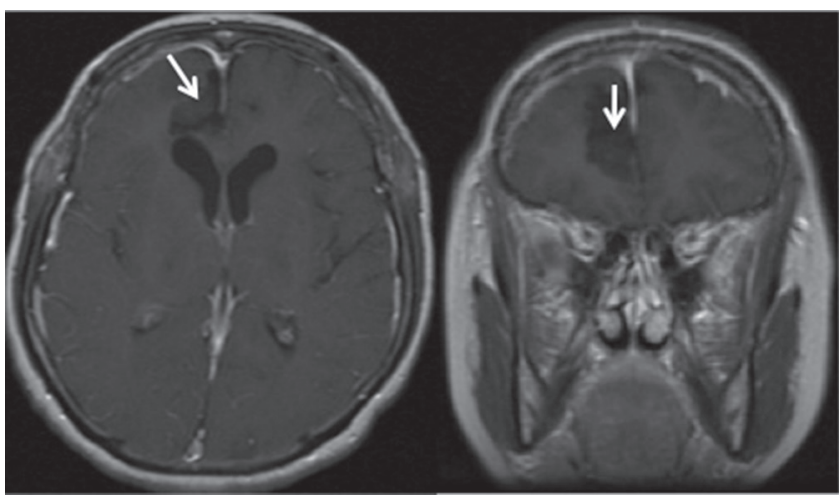

Fig. $2 \mathrm{~T}_{1}$-weighted post-contrast enhanced magnetic resonance (MR) images 3 months after the operation revealing a tumor in the hematoma cavity within the right frontal lobe (arrows).
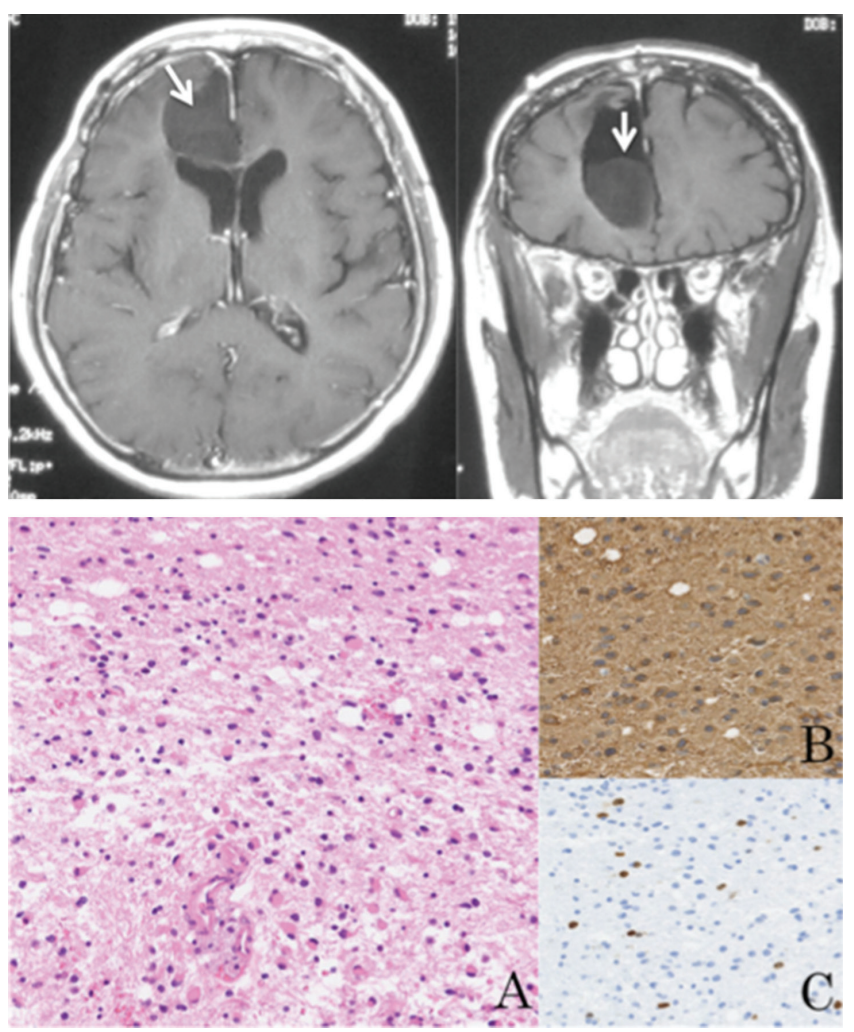

Fig. $3 T_{1}$-weighted post-contrast enhanced magnetic resonance (MR) images 3 months after the operation revealing a regrowth of the tumor in the hematoma cavity within the right frontal lobe (top, arrows). Histological examination of the resected tumor specimen showing the features of diffuse astrocytoma (bottom $\times 200$ ). A: hematoxylin and eosin stain (HE), B: glial fibrillary acidic protein (GFAP) positive, C: mindbomb homolog 1 (MIB-1) labeling index of $4-5 \%$.

The cause of hemorrhage in low-grade astrocytoma remains unclear. Even in pilocytic and pilomyxoid astrocytomas, which have higher vascularity, intratumoral hemorrhages are still rare. Possible causes may include 
abnormal vessels with thin walls, endothelial proliferation with vascular obliteration, invasion of a vessel wall by the tumor, tumor necrosis, increased venous pressure associated with raised intracranial pressure, or an encased aneurysm or vascular malformation. In the present case, histological examination of the specimens did not show vessel wall invasion, endothelial proliferation, or necrosis. We did not find any aneurysm or other vascular malformation in the histological examination of the specimen obtained from the hematoma. The bleeding may therefore have been a result of structural abnormalities in the tumor vessels and/or increased regional venous pressure associated with tumor expansion. Although rare, brain tumors, including low-grade astrocytoma, should be considered a possible cause of subcortical hemorrhage in patients without risk factors for intracranial hemorrhage.

\section{Conflicts of Interest Disclosure}

The authors declare that they have no conflict of interest. All authors who are the members of The Japan Neurosurgical Society (JNS) have registered online Self-reported COI Disclosure Statement Forms through the website for JNS members.

\section{References}

1) Bitoh S, Hasegawa H, Ohtsuki H, Obashi J, Fujiwara M, Sakurai M: Cerebral neoplasms initially presenting with massive intracerebral hemorrhage. Surg Neurol 22: 57-62, 1984

2) Golash A, Thorne J, West CG: Low grade pilocytic astrocytoma presenting as a spontaneous intracerebral haemorrhage in a child. Br J Neurosurg 12: 59-62, 1998

3) Lyons MK: Pilocytic astrocytoma with spontaneous intracranial hemorrhages in an elderly adult. Clin Neurol Neurosurg 109: 76-80, 2007

4) Lee CS, Huh JS, Sim KB, Kim YW: Cerebellar pilocytic astrocytoma presenting with intratumor bleeding, subarachnoid hemorrhage, and subdural hematoma. Childs Nerv Syst 25: 125-128, 2009

5) Shibahara I, Kanamori M, Kumabe T, Endo H, Sonoda Y, Ogawa Y, Watanabe M, Tominaga T: Hemorrhagic onset of pilocytic astrocytoma and pilomyxoid astrocytoma. Brain Tumor Pathol 26: 1-5, 2009

Address reprint requests to: Takao Nozaki, MD, PhD, Department of Neurosurgery, Hamamatsu University School of Medicine, 1-20-1 Handayama, Higashi-ku, Hamamatsu, Shizuoka 431-3192, Japan.

e-mail: tnozaki@hama-med.ac.jp 